University of Nebraska - Lincoln

DigitalCommons@University of Nebraska - Lincoln

2-1-1993

\title{
Harmonic Generation by an Intense Laser Pulse in Neutral and Ionized Gases
}

\author{
X. Liu \\ University of Michigan, Ann Arbor \\ Donald Umstadter \\ University of Nebraska-Lincoln, donald.umstadter@unl.edu \\ Eric Esarey \\ Beam Physics Branch, Naval Research Laboratory,Washington, DC, ehesarey@lbl.gov
}

A. Ting

Beam Physics Branch, Naval Research Laboratory, Washington, DC

Follow this and additional works at: https://digitalcommons.unl.edu/physicsumstadter

Part of the Physics Commons

Liu, X.; Umstadter, Donald; Esarey, Eric; and Ting, A., "Harmonic Generation by an Intense Laser Pulse in Neutral and lonized Gases" (1993). Donald Umstadter Publications. 42.

https://digitalcommons.unl.edu/physicsumstadter/42

This Article is brought to you for free and open access by the Research Papers in Physics and Astronomy at DigitalCommons@University of Nebraska - Lincoln. It has been accepted for inclusion in Donald Umstadter Publications by an authorized administrator of DigitalCommons@University of Nebraska - Lincoln. 


\title{
Harmonic Generation by an Intense Laser Pulse in Neutral and Ionized Gases
}

\author{
X. Liu, D. Umstadter, E. Esarey, and A. Ting
}

\begin{abstract}
Reported are the results of a harmonic generation experiment in a simple gas (hydrogen) using 1-ps, 1- $\mu$ m laser pulses with a range of intensities extending from below to far above the laser ionization saturation threshold. The scaling with intensity above saturation of the third harmonic generated by a single laser-pulse in a filled gas cell is observed to not fit with a simple model that takes into consideration volume ionization effects alone. In another experiment, a pump-probe type, an upper limit on the conversion efficiency of third harmonic generation in a preformed plasma is determined. It is found to be in agreement with the efficiency predicted by a relativistic harmonic generation theory.
\end{abstract}

\section{INTRODUCTION}

When a laser is focused to a relatively low intensity onto a gas [1]-[4], nonlinear motion of the bound electrons caused by the anharmonic atomic potential will generate frequencies at odd-harmonics of the laser frequency. At intensities exceeding the threshold for ionization, a plasma will be formed. A theoretical suggestion has been made recently that this ionization process, by generating nonlinear currents at twice the laser frequency, may also produce odd-harmonics [5]-[7]. At higher laser intensities, the free electrons in a plasma undergo nonlinear motion due to their relativistic mass change as their quiver velocity approaches the speed of light. This leads to the generation of harmonic radiation either incoherently, by laser-induced spontaneous radiation from individual electrons (Thomson scattering) [8], or coherently, by laser-driven plasma currents [9]-[11].

The goal of the experiment discussed in Section II was to conduct a study of these processes by focusing a laser pulse into a gas at intensities both below and far above the ionization saturation threshold, $I_{s}$. In this case, the laser will interact with the gas at the front of the pulse and the edges of the focus, and will form a plasma in the central region. Although the measured scaling of the harmonics with intensity are not found to fit with the current theoretical understanding, this comes as no surprise, given the complexity of the problem and the myriad of competing harmonic generation mechanisms. In order to study one of these mechanisms in isolation, we performed a pump-probe experiment, discussed in Section

Manuscript received June 28, 1992; revised October 13, 1992. This work was supported in part by the National Science Foundation Center for Ultrafast Optical Science, Contract \#PHY8920108 and the Office of Naval Research under Contract \#N00014-91-K-2005

X. Liu and D. Umstadter are with the Center for Ultrafast Optical Science, University of Michigan, Ann Arbor, MI 48109.

E. Esarey and A. Ting are with the Beam Physics Branch, Naval Research Laboratory, Washington, DC 20375-5000.

IEEE Log Number 9207463

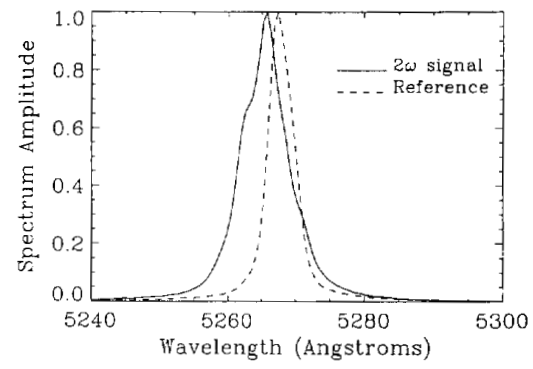

Fig. 1. Spectrum of SHG signal in the forward direction from hydrogen, $p=1$ Torr, with $\omega$ pulse energy $\sim 200 \mathrm{~mJ}$; the frequency-shift is due to rapid ionization of the gas. The reference spectrum was obtained from a doubling crystal.

III, in which an intense pulse interacted primarily with a preformed plasma. In so doing, we were able to establish an upper bound for the conversion efficiency by relativistic plasma harmonic generation at a particular density and laser intensity and compare it with that due to bound electrons.

\section{ATOMIC HARMONIC GENERATION}

In the first experiment, a single 1-ps pulse from a 1.053$\mu \mathrm{m} \mathrm{Nd:glass} \mathrm{laser} \mathrm{based} \mathrm{on} \mathrm{the} \mathrm{chirped} \mathrm{pulse} \mathrm{amplification}$ principle [12] was focused into a gas cell backfilled with hydrogen [13]. The spectrum of the SHG in the forward direction (Fig. 1) was recorded by a $1 / 3 \mathrm{~m}$ spectrometer coupled to an optical multichannel analyzer with an intensified linear photodiode array. The spectral resolution of the system was estimated to be $\sim 1.6 \AA$. The scalings of secondharmonic generation (SHG) and third-harmonic generation (THG), with gas pressure for fixed intensity, or with intensity for fixed pressure-shown in Fig. 2-were measured with a photomultiplier tube (PMT) and appropriate band-pass filters. The intensity at the focus was varied from a value that was much below, to one that was much above, the threshold for tunneling ionization. In the former case, the light pulse interacted with an atomic gas. In the latter case, the leading edge of the pulse rapidly ionized the gas and the remainder of the pulse interacted with a fully ionized plasma. The effect of any residual ionization would be to further enhance the generation of coherent harmonics, as discussed by Brunel [5]. However, with a laser pulse having a spatial Gaussian profile transverse to the propagation direction, there was always a region in the wings of the profile where the laser interacted with the neutral gas. As we will see later, it turned out that this region was actually responsible for our THG result. 

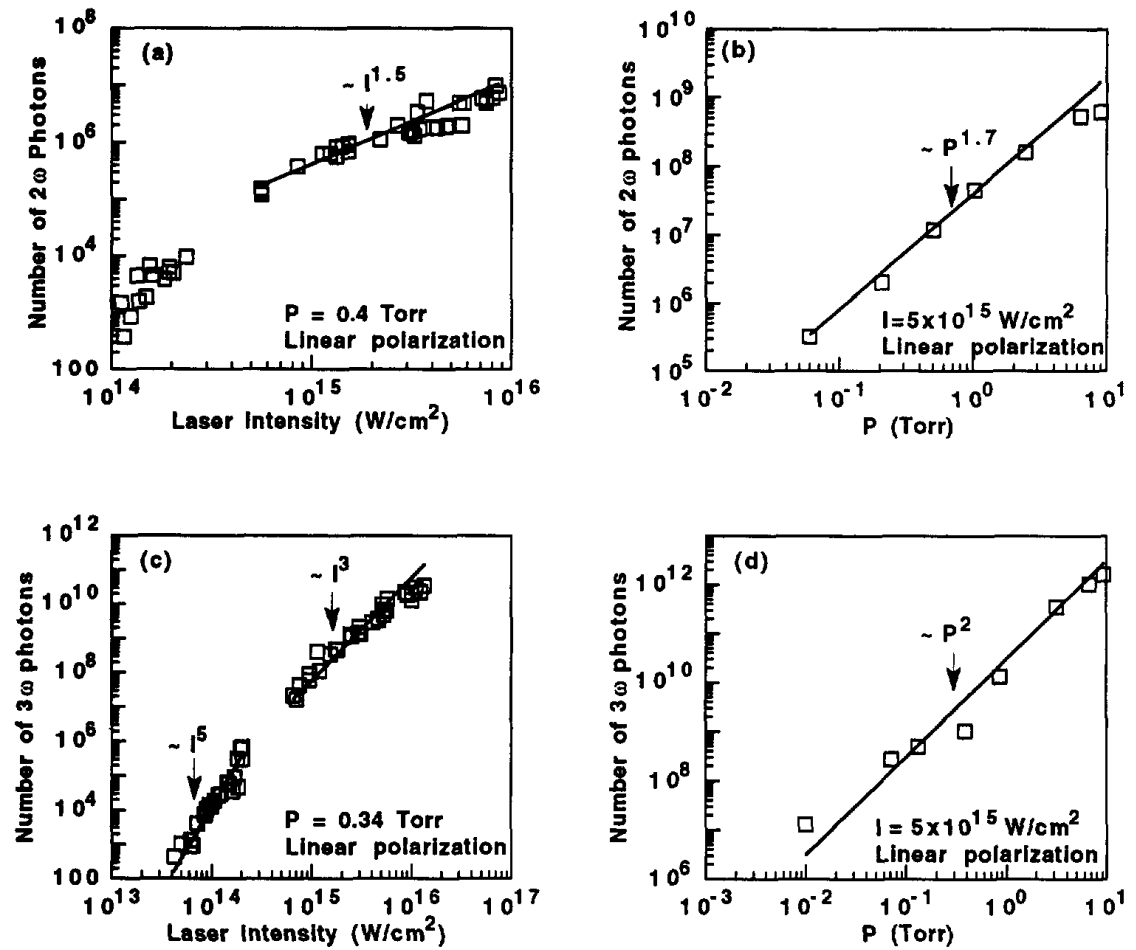

Fig. 2. Scaling of SHG and THG in a hydrogen plasma. (a) Below $I_{s} \sim 2 \times 10^{14} \mathrm{~W} / \mathrm{cm}^{2}$, the steep slope of SHG vs. laser intensity is characteristic of tunneling or multiphoton ionization of the gas. Above $I_{s}$, SHG scales as $P_{2} \propto I_{0}^{3 / 2}$, indicative of volume ionization effects. The gap in the data was solely due to the detection method. (b) The measured scaling of SHG with pressure, $P_{2} \sim p^{1.7}$. (c) Below $I_{s}$, the scaling of THG with intensity agrees with the theory of harmonic generation from bound electrons in a gas, $I_{0}^{7 / 2} \leq P_{3} \leq I_{0}^{5}$. Above $I_{3}$, the THG scales as $P_{3} \propto I_{0}^{3}$. (d) The measured scaling of THG with pressure, $P_{3} \sim p^{2}$.

The frequency shift and broadening of Fig. 1 may be explained by the rapid change of the index of refraction during the ionization of the gas [14]. The neutral gas density $n_{0}$ is assumed to be linearly proportional to the pressure through the ideal gas law, $p=n_{0} k T$. The electron density $n_{e}$ is assumed to be a large fraction $\left(n_{e} / n_{0}>0.1\right)$ of $n_{0}$. Calculations indicate that tunneling ionization is the dominant ionization mechanism. Ignoring collisional damping for the low pressures considered here, the index of refraction of the plasma is $n^{2}=1-\omega_{p}(t)^{2} / \omega^{2}=1-\left(4 \pi n_{e}(t) e^{2} / m\right) / \omega^{2}$, where the electron density $n_{e}(t)$ increases with time during the risetime of the laser pulse. The frequency shift due to this index change can be written as $\Delta \lambda / \lambda=-\Delta \omega / \omega=(z / c) d n / d t$, where $z$ is taken to be the Rayleigh range of the laser focus. For $\lambda=1 \mu \mathrm{m}, z \simeq 1 \mathrm{~cm}$, and supposing that $n_{e}(t)$ increases from 0 to $10^{16} \mathrm{~cm}^{-3}$ during the first $100 \mathrm{fs}$, then we would expect $\Delta \lambda \sim 1 \AA$, which is in approximate agreement with the observed frequency shift.

To obtain SHG in a medium with inversion symmetry in the electric-dipole approximation, the inversion symmetry must be broken by the action of the laser field. According to Bethune [15], the mechanisms leading to charge separation can either be the ponderomotive force of the laser, or the initial kinetic energy of the ejected electrons. The origin of SHG then is attributed to the third-order nonlinear mixing, via atomic nonlinear susceptibility $\left(\chi^{(3)}\right)$, between the laser field $\left(\omega=\omega_{0}\right)$ and the cylindrically symmetric static electric field $(\omega=0)$ created by the charge separation. The SHG polarization $\mathbf{P}(2 \omega)$ is proportional to the static field $E_{s}$. As expected, both SHG and THG were polarized along the same axis as the laser. However, whereas circularly polarized laser light was found to be more efficient in producing SHG than linear polarization, it produced no THG. As shown in Fig. 2(a), for a gas pressure of 0.4 torr, the scaling of SHG at low intensities $\left(I \leq 2 \times 10^{14} \mathrm{~W} / \mathrm{cm}^{2}\right)$ has a very steep slope. This slope at low intensities reflects the fact that the magnitude of the static field depends on the free electron density, which in turn depends on the laser intensity, either as a result of tunneling ionization $\left(n_{e} \sim\left(I_{s} / I\right)^{1 / 2} \exp \left[-\left(I_{s} / I\right)^{1 / 2}\right]\right)$, or multiphoton ionization $\left(n_{e} \sim I^{m}, m \sim 12\right.$ for hydrogen).

At higher laser intensities SHG scales as $P_{2} \propto I_{0}^{3 / 2}$. A similar change in scaling to one with $I_{0}^{3 / 2}$, described as saturation, has been observed in ion production [16]. This scaling is consistent with the expectations of theory. The exponent, $3 / 2$, arises from the increase in volume of the halo region of the laser focus, in which the gas is partially ionized [16]. The transition in the scaling in Fig. 2(a) indicates that the ionization saturation threshold intensity $\left(I_{s}\right)$, is seen to be $I_{s} \simeq 2 \times 10^{14} \mathrm{~W} / \mathrm{cm}^{2}$. The scaling of SHG with gas pressure, shown in Fig. 2, was determined by integrating the $2 \omega$ spectral curves and subtracting the background SHG. As 


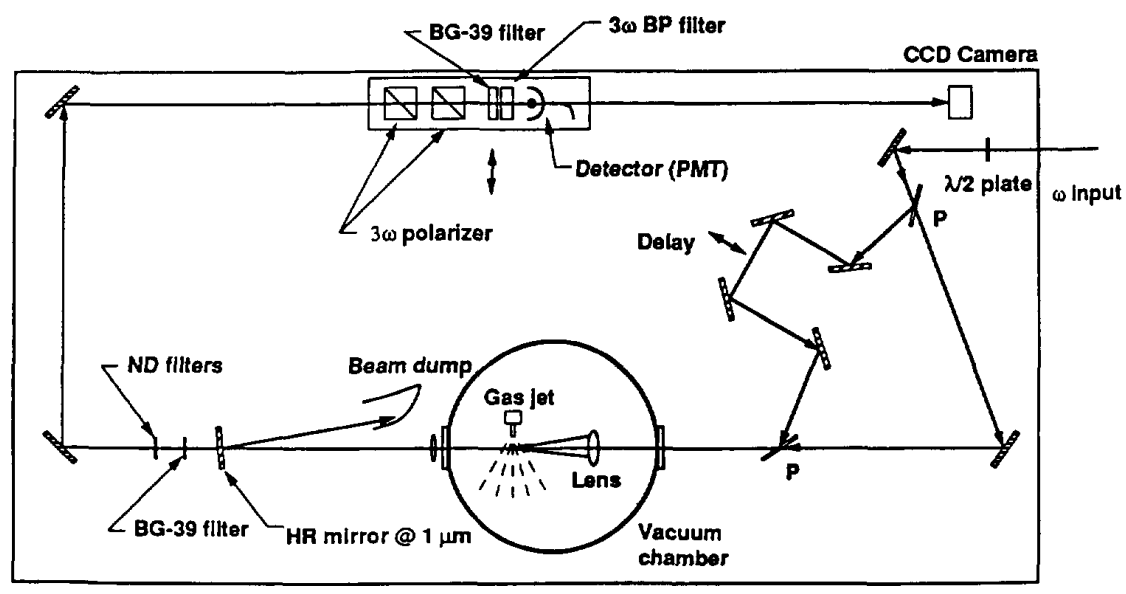

Fig. 3. Experimental pump-probe layout for THG in a plasma preformed by a laser pulse. The P-polarization beam transmitted through the polarizing beam splitters $\mathbf{P}$ was used as the pump pulse to produce the plasma. The reflected beam from $\mathbf{P}$, having S-polarization, was delayed by a variable delay line. BG-39 filters and a high reflection mirror at $1 \mu \mathrm{m}$ were used to cut the fundamental frequency laser after THG. THG by the pump pulse was rejected by two appropriately oriented $3 \omega$ polarizers by the property that $3 \omega$ has the same polarization as the fundamental. The digital CCD camera was used to align the overlap of the two beams when the detector assembly (the $3 \omega$ polarizers, filters and PMT) was removed.

is seen in Fig. 2(b) and (d), SHG scales at fixed intensity approximately as $p^{1.7}$ and THG as $p^{2}$ over the pressure range in the experiment. Theory predicts that SHG should scale as $p^{2}$. This, however, assumes a constant density and laser intensity, and from the discussion related to the frequency shift we see that the plasma density-and, of course, the intensity of the laser-change during the laser pulse. The predicted scaling is thus an upper bound. This may explain the discrepancy between the measured scaling of the SHG with pressure shown in Fig. 2, $P_{2} / P_{1} \sim p^{1.7}$,

THG also scales with laser intensity differently above $I_{s}$ than below it, as shown in Fig. 2(c), for a gas pressure equal to 0.34 torr: below, it scales as $I_{0}^{7 / 2} \leq P_{3} \leq I_{0}^{5}$, and above, $P_{3} \propto I_{0}^{3}$. Under our experimental conditions, with a confocal parameter less than the length of a positively dispersive medium, third harmonic generation is normally forbidden due to phase matching [17]. Our observation of THG is explained by invoking an argument that a laser-dependent nonlinear change in the refractive index (the Kerr effect) modifies the propagation and phase matching of the third harmonic [17], [18]. Above $I_{s}$, the scaling has yet to be predicted by a complete theory that accounts for phase matching, nonlinear index changes, nonlinear currents, and volume ionization effects. It appears, however, that the THG does not saturate at a level that would be expected from a simple model that takes into consideration volume ionization effects alone. In this case, a lower scaling - the same as is observed in SHG, $I_{0}^{3 / 2}$ - would be expected. In order to explain the results of similar experiments, others have speculated that a plasma may have been contributing to the harmonic production at intensities just above $I_{s}$ [19], [17]. In fact, the cubic scaling is precisely what is predicted by a theory for relativistic harmonic generation [9], [13] in a plasma. However, we can rule out this last possibility by the results of a pump-probe experiment, presented in the next section, in which a laser interacted only with a plasma.

\section{HaRmonic Generation IN Plasmas}

In order to study plasma harmonic generation by free electrons, without the competing effect of atomic harmonic generation by bound electrons, a preformed plasma is required. This was accomplished by means of a pump-probe type configuration, as shown in Fig. 3. The incoming laser pulse was split in two with a polarizing beam splitter. The intensity ratio of the two beams could be varied by the use of a halfwave plate. The weaker beam, used to study THG in plasmas, was sent through a delay line. The two beams were combined to propagate collinearly by a second polarizing beam splitter, and brought to a common focus with the same focusing lens. Their spatial overlap transverse to the direction of propagation was determined to an accuracy of better than $2 \mu \mathrm{m}$, or $5 \%$ of their focal diameters, by imaging their foci with a lens with a magnification factor of twenty and a digital CCD camera. The two laser pulses, each of 1 ps duration, were focused to intensities of $5 \times 10^{16} \mathrm{~W} / \mathrm{cm}^{2}$ and $1 \times 10^{16} \mathrm{~W} / \mathrm{cm}^{2}$, respectively, onto a pulsed molecular beam of hydrogen. This value for the intensity of the second pulse corresponds to a normalized vector potential of $a=0.1$, where $a=e E_{0} / m c \omega$. The gas density of the molecular beam in the laser focal volume could be varied up to $\sim 5 \times 10^{17} \mathrm{~cm}^{-3}$ ( $\sim 15$ torr). With this configuration, the first pulse created a plasma in advance of the arrival of the second pulse. Hydrogen was chosen as the gas because of its single-ionization stage. It is well known [1]-[4], and verified in our experiment, that for linear polarization, the direction of polarization of the third harmonic is along that of the laser. Since the two incident pulses were polarized orthogonally, their third harmonics were also polarized orthogonally. Therefore, THG by the second pulse, which interacts only with a plasma, was separated from THG by the first pulse, which interacts with the gas, by the use of a polarizer (analyzer).

We found that with less than a 10-ps delay between the two pulses (much less than the recombination time at this density, 
$n_{e} \sim 10^{17} \mathrm{~cm}^{-3}$ ), THG by the second pulse was at least $10^{5}$ less than in the case when the first pulse was blocked, i.e., no preformed plasma was produced. In other words, for our laser parameters, the power conversion efficiency for harmonic generation in a plasma formed only of free electrons and bare nuclei is at least five orders of magnitude less than that in a neutral gas. This translates into an upper bound on the conversion efficiency to third-harmonic generation by the mechanism of relativistic harmonic generation of $\eta_{3} \leq$ $10^{-13}$. This is consistent with a value predicted by theory of $\eta_{3} \leq 10^{-14}[20]-[22]$. The same theory predicts that a higher efficiency should be obtained at higher plasma density and laser intensity. The determination of the upper bound for THG in the plasma was mainly limited by the extinction ratio of the analyzer in front of the detector, since there was always some leakage of THG of the orthogonal polarization by the first pulse. The minimum detection level for THG in the plasma is limited by this leakage. With this limit in mind, we saw no THG in the plasma.

\section{DISCUSSION AND CONCLUSION}

We have measured experimentally the SHG and THG scaling with laser intensities below and above the ionization saturation intensity, $I_{s}$. For $I>I_{s}$, SHG scaling can be attributed to ionization saturation. However, THG scaling is not explained adequately by ionization saturation alone. Instead, a combination of volume ionization effects, the Kerr effect, or nonlinear currents from ionization [5], may be required for an explanation.

The pump-probe THG experiment is, to our knowledge, the first attempt to observe THG in a preformed plasma. Although we were only only able to determine an upper limit for the THG efficiency, the experiment nevertheless demonstrates the importance of collective effects in a plasma. In fact, if we ignore the plasma effect and treat the free electrons as individual radiators, the relativistic harmonic generation mechanism would produce a third harmonic signal far above our detection limit [13]. In this case, the THG efficiency would be given by $\eta_{3}=P_{3} / P_{1}=\left[(\pi / 8)\left(\lambda^{2} / \lambda_{p}^{2}\right)(L / \lambda) a^{2}\right]^{2}$, where $\lambda$ is the laser wavelength, $\lambda_{p}$ the plasma wavelength $\left(\lambda_{p}=\right.$ $2 \pi c / \omega_{p}, \omega_{p}$ is the plasma frequency), $L$ is the laser-plasma interaction length, roughly twice the Rayleigh length, and $a_{o}$ is the normalized vector potential. Using our laser parameters we would get $\eta_{3} \sim 10^{-6}$-many orders of magnitude higher than our experimentally determined $\eta_{3} \leq 10^{-13}$. Only when we take both the plasma effect and phase matching into consideration is the relativistic harmonic generation theory consistent with the experimental observation, as indicated above.

In order to verify the relativistic harmonics theory by such a pump-probe type experiment, one will have to have both a higher laser intensity and better discrimination against the harmonics generated by the first pulse. In addition, boundelectron harmonic generation by the second pulse at the edge of the focal volume can also introduce ambiguities to the experiment. This may be overcome by increasing the intensity ratio of the first pulse to the second. Other experimental configurations are currently being considered, including the use of a streak camera to discriminate the harmonic signals of the two laser pulses.

\section{ACKNOWLEDGMENT}

The authors thank C. Y. Chien and S. Coe for helpful assistance with the laser operation, and G. Mourou for many encouraging discussions.

\section{REFERENCES}

[1] J. F. Ward and G. H. C. New, "Optical third harmonic generation in gases by a focussed laser beam," Phys. Rev., vol. 185, p. 57, 1969.

[2] A. McPherson, G. Gibson, H. Jara, U. Johann, T. S. Luk, I. A. McIntyre, K. Boyer, and C. K. Rhodes, "Studies of multiphoton production of vacuum-ultraviolet radiation in the rare gases," J. Opt. Soc. Amer. B, vol. 4 , p. 595, 1987.

[3] K. C. Kulander and B. W. Shore, "Generation of optical harmonics by intense pulses of laser radiation. II. Single-atom spectrum for xenon," J. Opt. Soc. Amer. B, vol. 7, pp. 502-508, 1990.

[4] L. A. Lompre', A. L'Huillier, M. Ferray, P. Monot, G. Mainfray, and C. Manus, "High-order harmonic generation in xenon: Intensity and propagation effects," J. Opt. Soc. Amer. B, vol. 7, p. 754, 1990.

[5] F. Brunel, "Harmonic generation due to plasma effects in a gas undergoing multiphoton ionization in the high-intensity limit," $J . O p t$. Soc. Amer. B, vol. 7, p. 521, 1990.

[6] W. P. Leemans, C. E. Clayton, W. B. Mori, K. A. Marsh, A. Dyson, and C. Joshi, "Plasma physics aspects of tunnel-ionized gases," Phys. Rev. Lett., vol. 68, pp. 321-324, 1992.

[7] W. P. Leemans, C. E. Clayton, W. B. Mori, K. A. Marsh, P. K. Kaw, A. Dyson, C. Joshi, and J. M. Wallace, "Experiments and simulations of tunnel-ionized plasmas," Phys. Rev. A 46, pp. 1091-1105, 1992.

[8] E. S. Sarachik and G. T. Schappert, "Classical theory of the scattering of intense laser radiation by free electrons," Phys. Rev. D, vol. 1, p. 2738, 1970.

[9] P. Sprangle, E. Esarey, and A. Ting, "Nonlinear theory of intense laser-plasma interactions," Phys. Rev. Lett., vol. 64, p. 2011, 1990.

[10] A. Ting, E. Esarey, and P. Sprangle, "Nonlinear wake-field generation and relativistic focusing of intense laser pulses in plasmas," Phys. Fluids $B$, vol. 2, p. $1390,1990$.

[11] P. Sprangle, E. Esarey, and A. Ting, "Nonlinear interaction of intense laser pulses in plasmas," Phys. Rev. A, vol. 41, p. 4463, 1990.

[12] G. Mourou and D. Umstadter, "Development and applications of compact high-intensity lasers," Phys. Fluids B, vol. 7, p. 2374, 1992.

[13] X. Liu, D. Umstadter, J. S. Coe, C. Y. Chien, E. Esarey, and P. Sprangle, "Harmonic generation by an intense picosecond laser in an underdense plasma," in OSA Proceedings on Short Wavelength Coherent Radiation: Generation and Applications, 1991, P. Bucksbaum and N. Ceglio, Eds. Washington, D.C.: Optical Society of America, 1989, 11, pp. 7-11.

[14] W. M. Wood, G. Focht, and M. C. Downer, "Tight focusing and blue shifting of millijoule femtosecond pulses from a conical axicon amplifier," Opt. Lett., vol. 13, p. 984, 1988.

[15] D. S. Bethune, "Optical second-harmonic generation in atomic vapors with focussed beams," Phys. Rev. A 23 , p. 3139, 1981.

[16] M. R. Cervenan, R. H. C. Chan, and N. R. Isenor, "Three photon ionization of alkali atoms at the ruby laser wavelength," Can. J. Phys., vol. 53 , p. 1573,1975 .

[17] A. L'Huillier, L. A. Lompre', M. Ferray, X., F. Li, G. Mainfray and C. Manus, "Third-harmonic generation in Xenon in a pulsed jet and a gas cell," Europhys. Lett. 5, 601(1988).

[18] M. Malcuit, R. W. Boyd, W. V. Davis, and K. Rzazewski, "Anomalies in optical harmonic generation using high-intensity laser radiation," Phys Rev. A, vol, 41, p. 3822, 1990.

[19] S. Augst, D. D. Meyerhofer, C. I. Moore, and J. Peatross, "Tunneling ionization and harmonic generation in krypton gas using a high-intensity, 1- $\mu \mathrm{m}, 1-\mathrm{ps}$ laser," in Proceedings of SPIE OE/LASE '90 1229, 152 (Los Angeles), Jan. 1990.

[20] E. Esarey, A. Ting, P. Sprangle, D. Umstadter and X. Liu, "Nonlinear analysis of relativistic harmonic generation by intense lasers in plasmas," IEEE Trans. Plasma Sci., vol. 21, pp. 95-104, Feb. 1993.

[21] J. M. Rax and N. J. Fisch, "Phase matched third harmonic generation in a plasma," IEEE Trans. Plasma Sci., vol. 21, pp. 105-109, Feb. 1993.

[22] W. B. Mori, C. D. Decker, and W. P. Leemans, "Relativistic harmonic content of nonlinear electromagnetic waves in underdense plasmas," IEEE Trans. Plasma Sci., vol. 21, pp. 110-119, 1993. 
D. Umstadter, photograph and biography not available at the time of A. Ting, photograph and biography not available at the time of publication. publication. 